\title{
Aldose reductase (EC 1.1.1.21) activity and reduced-glutathione content in lenses of diabetic sand rats (Psammomys obesus) fed with acarbose
}

\author{
BY ESTER COHEN-MELAMED ${ }^{1}$, A. NYSKA ${ }^{2}$, A. POLLACK ${ }^{3}$ AND Z. MADAR ${ }^{1}$ \\ ${ }^{1}$ Department of Biochemistry, Food Science and Nutrition, Faculty of Agriculture, The Hebrew \\ University of Jerusalem, PO Box 12, Rehovot 76100, Israel \\ ${ }^{2}$ Kimron Veterinary Institute, Beit Dagan, Israel and Koret School of Veterinary Medicine, \\ The Hebrew University of Jerusalem, Israel \\ ${ }^{3}$ Kaplan Hospital, Rehovot, Israel
}

(Received 3 January 1995 - Revised 7 March 1995 - Accepted 17 March 1995)

\begin{abstract}
The effects of acarbose on cataract development, lens aldose reductase (EC 1.1.1.21) activity and lenticular reduced-glutathione content in diabetic sand rats (Psammomys obesus) were determined. Diabetic sand rats (diet-induced) were fed on diets with or without acarbose $(0.4 \mathrm{~g} / \mathrm{kg})$ for $39 \mathrm{~d}$. Daily plasma glucose, cataract incidence, aldose reductase and glutathione content were evaluated. After 19 d on acarbose, daily plasma glucose profile was significantly reduced compared with that of sand rats not receiving acarbose. Cataract incidence was markedly lower in sand rats treated with acarbose. After $20 \mathrm{~d}$, cataracts had developed in $90 \%$ of the animals fed without acarbose, whereas none was observed in sand rats fed with acarbose. After $37 \mathrm{~d}$ acarbose treatment the incidence of cataracts reached only $30 \%$. Compared with untreated animals, lens aldose reductase activity was significantly lower in sand rats fed with acarbose for $39 \mathrm{~d}(7.6$ (SE 0.78) v. 3.5 (SE 0.55) $\mu \mathrm{mol}$ NADPH/mg protein per min respectively, $P<0.001$ ). Concomitantly, significantly higher lenticular protein and reduced-glutathione contents (90 (SE 23) v. 240 (SE 23.5) $\mu \mathrm{g} / \mathrm{mg}$ tissue respectively, $P<0.001$ and 369 (SE 48.6) v. 645 (SE 71-1) $\mu \mathrm{g} / \mathrm{mg}$ tissue respectively, $P<0.001$ ) were found. These results suggest that decreasing hyperglycaemia, accompanied by lower aldose reductase activity obtained by acarbose, led to a significant preventive effect on cataract development in sand rats.
\end{abstract}

Acarbose: Cataract: Aldose reductase: Sand rats

The association between diabetes and its long-term complications, such as cataracts, has been well established (Zimmerman, 1992; Diabetes Control and Complication Trial Research Group, 1993). Thus, drugs treating the short-term complications of diabetes (i.e. uncontrolled plasma glucose levels and their immediate effects) should also be capable of preventing or delaying the development of long-term complications. Evidence strongly suggests the long-term complications of diabetes to be the result of hyperglycaemia (Zimmerman, 1992). The hyperglycaemic condition in experimental models is known to cause lenticular changes (Kador et al. 1986; Kinoshita, 1986). These changes, including increased sorbitol levels, altered membrane permeability, loss of reduced glutathione (GSH), decreased amino acid levels and decreased protein synthesis, eventually lead to cataract formation. Experimental evidence strongly indicates that aldose reductase (AR; $E C$ 1.1.1.21) initiates the process leading to sugar cataracts via the production of sorbitol (Kador et al. 1986; Kinoshita, 1986). AR utilizes NADPH to reduce glucose to sorbitol while, in the next step, sorbitol dehydrogenase $(E C 1.1 .1 .14)$ utilizes $\mathrm{NAD}^{+}$to oxidize sorbitol to fructose. AR inhibitor was effective in preventing cataract development (Kato 
et al. 1990). Glucose entry into the lens is not modulated by either insulin or glycolysis. Hence, hyperglycaemia leads to increased glucose in this tissue and increased AR activity, whereas sorbitol dehydrogenase activity decreases. This shift in enzyme activity, combined with increased available lenticular glucose levels, favours sorbitol accumulation, which leads to cataract formation (Kador et al. 1986; Kinoshita, 1986).

Despite the evidence suggesting that long-term complications of diabetes result from hyperglycaemia, available information indicates that standard treatment methods do little to reduce the prevalence of these late-forming complications (Zimmerman, 1992). Acarbose is a polysaccharide synthesized by a microbial (Actinomyces) culture. Acarbose, an $\alpha-$ glucosidase ( $E C$ 3 2.1 .20 ) inhibitor, is not absorbed into the systemic circulation and is a competitive inhibitor of dextrinase $(E C 3.2 .1 .10,48)$, sucrase $(E C 3.2 .1 .48)$, isomaltase $(E C$ 3.2.1 10) and glucoamylase (EC 3.2.1.3) (Bischoff, 1993). This drug has been found to be effective in controlling plasma glucose levels (Clissold \& Edwards, 1988; Taylor, 1991; Bischoff, 1993) and thus has the potential to reduce late complications in diabetes (Zimmerman, 1992; Bischoff, 1993).

The present work evaluated the effect of acarbose on the development of cataracts in sand rats (Psammomys obesus) as a model for type 2 diabetes. In its natural environment the sand rat feeds exclusively on succulent plants with low energy value and high salt content, and demonstrates normal blood glucose levels. However, long-term feeding of a 'high'-energy (synthetic chow) diet results in obesity and a diabetes syndrome (type 2) which includes insulin resistance, hyperinsulinaemia, markedly decreased glucose tolerance, hyperglycaemia and cataracts (Marquie et al. 1984). The sand rats used in the present study were from a specially defined diabetic (type 2) line, a colony developed at the Hebrew University of Jerusalem, Israel (Kalman et al. 1993).

The present study aimed at evaluating the effect of acarbose on plasma glucose profiles, cataract development and histological changes in the lenses of sand rats. Its effect on lens aldose reductase activity and the contents of lenticular protein and GSH were measured as well.

\section{MATERIALS AND METHODS}

\section{Animals}

Twenty weanling male sand rats (The Hebrew University of Jerusalem, Israeli strain, diabetic type 2 line) with an initial body weight of 109 (SE 9) g were divided into two groups of ten animals each with equal mean body weights (109 (SE 11) and 109 (SE 13) g respectively). The animals were housed in individual cages maintained at $22 \pm 1^{\circ}$ with a 12:12 h light-dark cycle: lights on from 06.00 to 18.00 hours. Guidelines issued by The Hebrew University of Jerusalem, Israel, for handling the animals were observed.

\section{Diets}

During a $39 \mathrm{~d}$ period the sand rats were fed on diets served as chow, composed of $(\mathrm{g} / \mathrm{kg})$ : soyabean protein 200 , maize starch 650 , soyabean oil 50 , dietary fibre 40 , minerals 40 and vitamins 20. They were maintained on the described diet (control group) or on a similar diet containing $0.4 \mathrm{~g}$ acarbose (BAY G 5421; Bayer, Germany)/kg (experimental group). During the experiment, all animals were allowed $a d$ lib. access to both feed and water.

\section{Plasma glucose levels}

Blood samples were collected from the tail on day 19 of the experiment in heparinized tubes containing $\mathrm{NaF}$, at $08.00,12.30,16.30$ and 21.00 hours, to determine daily plasma glucose profiles. The plasma samples were assayed for glucose by the glucose oxidase $(E C 1.1 .3 .4)$ 

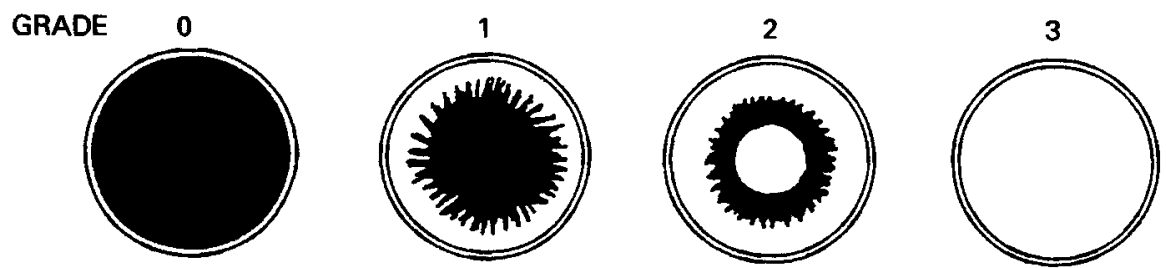

Fig. 1. Clinical grading criteria of cataracts: grade 0, lens completely clear; grade 1, opaque areas at periphery, vascular features of the retina still visible; grade 2, widespread distribution of opaque areas within the lens, vascular features of the retina obscured; grade 3, lens totally opaque, cataract visible as a dense white mass to the naked eye.

method (Morrison, 1972) using a Beckman glucose analyser (Beckman Instruments Inc., Fullerton, CA, USA).

\section{Classification of cataracts}

The lenses were evaluated (without anaesthesia) using a hand-held ophthalmoscope in a masked fashion. Pupils were dilated by a single topical application of tropicamide $(5 \mathrm{~g} / \mathrm{l})$ and phenylephrine $(100 \mathrm{~g} / 1)$. Cataracts were clinically classified as follows: grade 0 , lens completely clear; grade 1, opaque areas at lens periphery, vascular retinal features still visible; grade 2, widespread distribution of opaque areas within the lens, vascular retinal features obscured; grade 3, lens totally opaque, cataract visible to the naked eye as a dense white mass (Fig. 1). Cataract grading was evaluated at random in 'blind' fashion without knowledge of which treatment was given to the rats.

Incidence of cataract appearance was expressed as percentage of sand rats developing cataracts in one or both eyes. A lens reaching grade 1 was regarded as having developed a cataract. The mean grade was calculated as the average of grades in all eyes in each group.

An additional separate experiment was performed for histological evaluation. This time sand rats were maintained on diets with or without acarbose for $26 \mathrm{~d}$. Enucleated globes were fixed by immersing in Davidson's fluid for $10 \mathrm{~h}$ and then stored in ethanol $(700 \mathrm{ml} / \mathrm{l})$ until trimming. The globes were trimmed sagittally on the meridional plane at the optic disk level. Both hemispheres were embedded in paraffin wax and $5-\mu \mathrm{m}$-thick sections were prepared and stained with haematoxylin and eosin (HE) (Bancroft \& Stevenes, 1972). Histological evaluation was carried out by an experienced pathologist, in a 'blind' fashion to avoid bias (i.e. the pathologist did not have any knowledge of the animals from which the tissue originated. The original labels on the slides of the tissue were masked).

The pathologic description took into consideration factors such as localization, stage of development and histological features. On histological evaluation, cataract involvement of the lens was expressed as percentage areas by using a scale of five grades in which grade 0 indicated no cataractous changes, grade 1 indicated that up to $25 \%$, grade 2 indicated that up to $50 \%$, grade 3 indicated that up to $75 \%$ and grade 4 indicated that up to $100 \%$ of the lens area was affected. Clinical cataract grade 1 is consistent with histological cataract grades 1 and 2, clinical grades 2 and 3 correspond to histological grades 3 and 4 .

\section{Aldose reductase activity and protein content in the lens}

AR activity was assayed according to the method of Kinoshita (1986) with modifications, some described by Halder \& Crabbe (1984); lens tissue from each sand rat was used. After 39 experimental days, sand rats were anaesthetized by injection of Nembutal $(80 \mathrm{mg} / \mathrm{kg}$ body weight). Then, each right lens was removed rapidly (on ice), weighed and homogenized in a Heidolph homogenizer (Heidolph Co., Kelheim, Germany) for $1 \mathrm{~min}$ in five volumes of ice-cold $50 \mathrm{~mm}$-potassium-phosphate buffer, $\mathrm{pH} 7 \cdot 2$, containing $5 \mathrm{mmol} 2$-mercaptoethanol/1. All biochemical analyses were carried out in each rat. The homogenate was 
centrifuged at $12300 \mathrm{~g}$ for $6 \mathrm{~min}$ at $4^{\circ}$ and the supernatant fraction was stored at $-20^{\circ}$. The frozen supernatant fraction was used for determination of AR activity and protein content in the lens.

AR activity was determined by monitoring the oxidation of NADPH at $340 \mathrm{~nm}$ using a Milton Roy spectrophotometer (Spectronic 601, NY, USA). The assay mixture was

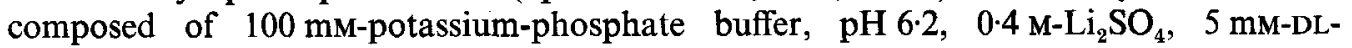
glyceraldehyde and $50 \mu \mathrm{M}-\mathrm{NADPH}$ (freshly made) and used immediately. The total volume, including lens supernatant fraction, was $1.0 \mathrm{ml}$. The reaction, carried out at $25^{\circ}$, was initiated by addition of the supernatant fraction containing the enzyme and was allowed to proceed for 6 min. A blank was run concurrently, without supernatant fraction for non-specific NADPH oxidation. The blank was subtracted from the reaction which included the lens homogenate. Protein content was determined according to the method of Bradford (1976). Bovine serum albumin was used as a standard.

\section{Reduced-glutathione content in the lens}

After the sand rats had been anaesthetized by Nembutal injection ( $80 \mathrm{mg} / \mathrm{kg}$ body weight), the left lens was rapidly removed (on ice), immediately placed in liquid $\mathrm{N}_{2}$ and stored in a firmly sealed plastic tube at $-70^{\circ}$ until assay. GSH content in the lens was determined by the fluorophotometric method of Hissin \& Hilf (1976) with slight modifications, using $o$ phthalaldehyde (OPT) as a fluorescent reagent. This method exploits the reaction of GSH with OPT at pH 8.

Frozen lenses were weighed and homogenized on ice in a Heidolph homogenizer for $1 \mathrm{~min}$ in 16 volumes of $0.1 \mathrm{M}-\mathrm{Na}_{3} \mathrm{PO}_{4}-0.005 \mathrm{M}$-EDTA buffer (pH 8) and 5 volumes of $\mathrm{H}_{3} \mathrm{PO}_{3}(250 \mathrm{ml} / 1)$. The total homogenate was centrifuged at $4^{\circ}$ at $12300 \mathrm{~g}$ for $30 \mathrm{~min}$ to obtain the supernatant fraction for GSH assay. The supernatant fraction was diluted with four volumes of the phosphate-EDTA buffer, pH 8 . The final assay mixture $(2.0 \mathrm{ml})$ contained $100 \mu$ l of the diluted tissue supernatant fraction, $1.8 \mathrm{ml}$ phosphate-EDTA buffer and $100 \mu \mathrm{l}$ OPT solution containing $100 \mu \mathrm{g}$ OPT (Sigma, St Louis, MO, USA). After thorough mixing and subsequent incubation at room temperature for $15 \mathrm{~min}$, fluorescence at $420 \mathrm{~nm}$ was determined with activation at $350 \mathrm{~nm}$, using a JASCO FP-550 spectrofluorometer (Spectroscopic Co. Ltd, Tokyo, Japan) and compared with a GSH standard curve. GSH (Sigma) was prepared daily in the phosphate-EDTA buffer and kept on ice until used. OPT solution was prepared daily in reagent-grade absolute methanol just before use.

\section{Statistics}

All values are means with their standard errors. Significance of differences was determined by Student's $t$ test. For cataract grades, comparisons were made by the Wilcoxon test.

\section{RESULTS}

The effect of acarbose on daily plasma glucose profiles in sand rats is shown in Table 1. Acarbose treatment for $19 \mathrm{~d}$ significantly reduced daily plasma glucose levels compared with those of sand rats given no acarbose.

Cataract development was observed throughout the experiment. As shown in Fig. 2, the incidence of cataracts was markedly lower in sand rats treated with acarbose. After $20 \mathrm{~d}$, cataracts developed in $90 \%$ of the animals fed on a diet without acarbose, while the group receiving acarbose was cataract-free. After $37 \mathrm{~d}$ acarbose treatment a moderate increase in cataract appearance was noted, reaching only $30 \%$ (Fig. 2). The mean grade of cataract observed, expressed as described in Fig. 1, was significantly lower in sand rats treated with acarbose (Fig. 3). After $20 \mathrm{~d}$ the animals fed on the acarbose-free diet had a mean grade of $2 \cdot 1$ whereas in sand rats treated with acarbose the mean grade of cataract was 0 , i.e. the 
Table 1. Daily plasma glucose profile in sand rats (Psammomys obesus) treated or untreated with acarbose for $19 d$

(Mean values with their standard errors)

\begin{tabular}{|c|c|c|c|c|c|c|c|c|}
\hline \multirow{3}{*}{$\begin{array}{l}\text { Time of day... } \\
\text { Treatment }\end{array}$} & \multicolumn{8}{|c|}{ Plasma glucose levels (mmol/l) } \\
\hline & \multicolumn{2}{|c|}{08.00} & \multicolumn{2}{|c|}{12.30} & \multicolumn{2}{|c|}{16.30} & \multicolumn{2}{|c|}{21.00} \\
\hline & Mean & $\mathrm{SE}$ & Mean & $\mathrm{SE}$ & Mean & SE & Mean & $\mathrm{SE}$ \\
\hline (-) Acarbose & \multicolumn{2}{|c|}{$(n 8)$} & \multicolumn{2}{|c|}{${ }_{(n 8)}^{16 \cdot 5}$} & \multicolumn{2}{|c|}{${ }^{100}(n 7)^{20}$} & \multicolumn{2}{|c|}{$(n 7)$} \\
\hline (+) Acarbose & ${ }_{(n}^{11 \cdot 2 *}$ & $1 \cdot 7$ & $11 \cdot 4$ & $2 \cdot 1$ & ${ }_{(n}^{10 \cdot 1 *}$ & $1 \cdot 7$ & $9 \cdot 6^{*}$ & 1.8 \\
\hline
\end{tabular}

* Mean values were significantly different from those for the group without acarbose, $P<0.05$.

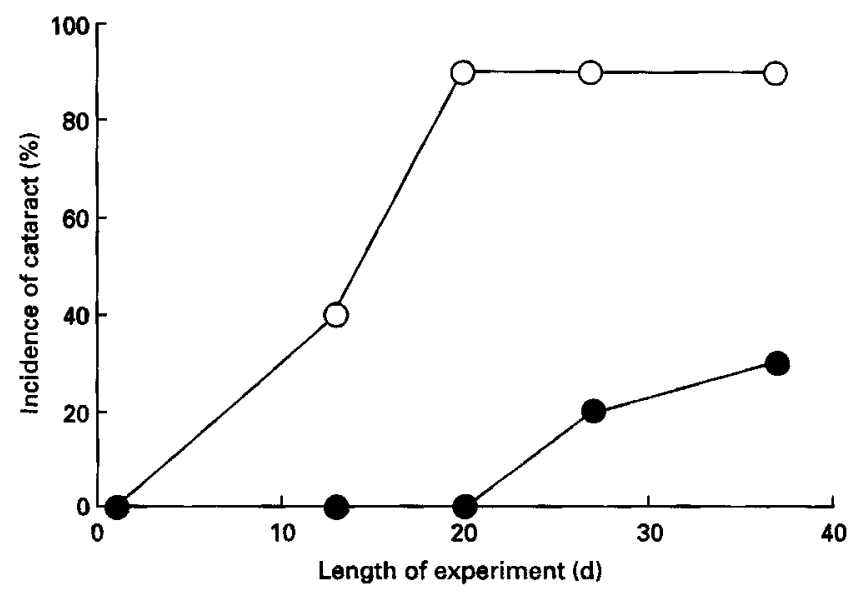

Fig. 2. Incidence of cataracts in sand rats (Psammomys obesus) fed with acarbose $(0.4 \mathrm{~g} / \mathrm{kg}$ diet; 0$)$ or untreated $(O)$. Values are means for ten rats per dietary group.

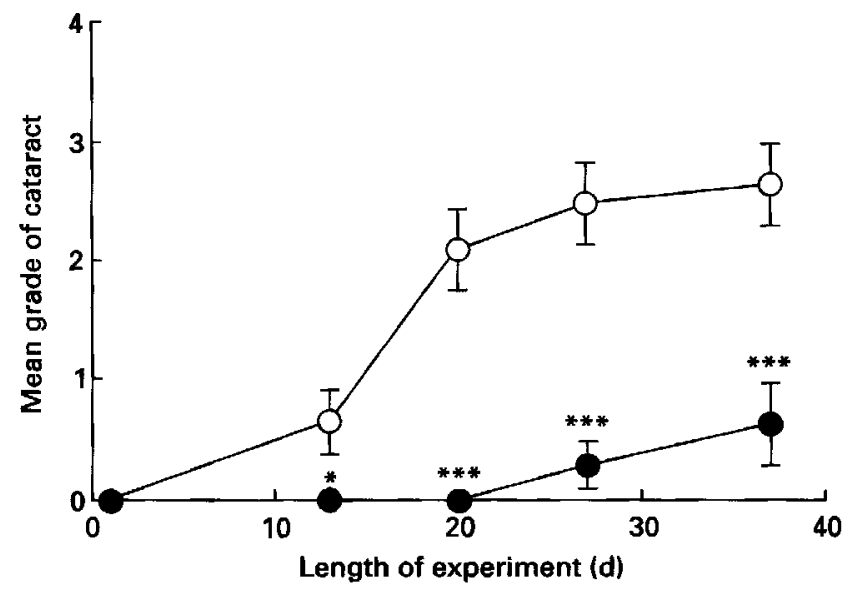

Fig. 3. Grade of cataract in sand rats (Psammomys obesus) fed with acarbose $(0.4 \mathrm{~g} / \mathrm{kg}$ diet; 0$)$ or untreated $(O)$. Values are means for ten rats per dietary group, with their standard errors indicated by vertical bars. Mean values were significantly different from those for the untreated group: ${ }^{*} P<0.05,{ }^{* * *} P<0.001$. 

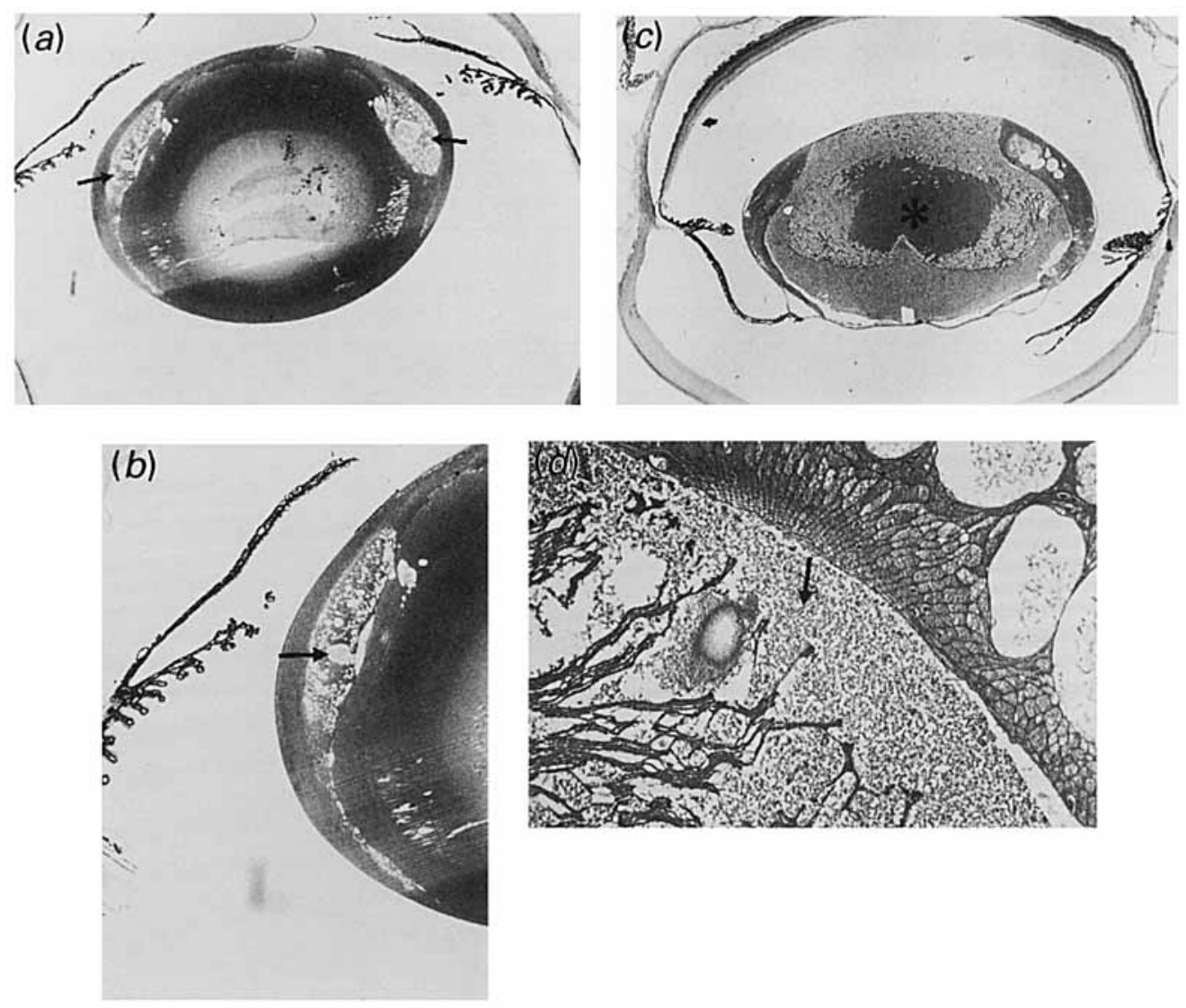

Fig. 4. (a), Low magnification view of a histological section of the eye of a sand rat (Psammomys obesus) with a grade 1 cataract ( $25 \%$ area of lens with cataractous changes). The arrows indicate a bilateral cortical equatorial cataract; (haematoxylin and eosin stain $(H E) \times 50)$. $(b)$, Higher magnification view of a histological section of the eye of a sand rat with a grade 1 cataract. The arrow indicates a cortical equatorial cataract due to fibre fragmentation and disintegration; $(\mathrm{HE} \times 200)$. $(c)$, Low magnification view of a histological section of the eye of a sand rat with a grade 3 cataract ( $75 \%$ of the lens area exhibiting cataractous changes): note the circumferential cataractous modifications. Only lens nucleus $(*)$ remains intact $(\mathrm{HE} \times 50)$. $(d)$, Higher magnification view of a histological section of the eye of a sand rat with a grade 3 cataract. The arrow indicates fibre disintegration associated with formation of lakes of granular lytic proteinaceous material $(\mathrm{HE} \times 200)$.

group was cataract-free $(P<0.001)$. Following $37 \mathrm{~d}$ feeding with acarbose the mean grade of cataract remained significantly lower than in the group fed without acarbose $(0.65$ (SE 0.35) v. 2.67 (SE 0.33) respectively, $P<0.001$ ).

Histological observations of the lenses are shown in Fig. 4. Grade 1 cataract involved both sides of the lens, localizing to the outer, lateral and medial cortical layers. With advancing degree the cataracterous changes progressed to inner layers and circumferentially. Finally, grade 4 , the inner nuclear material, was found surrounded by disintegrated liquefied material.

Biochemical variables involved in cataract development were measured after $39 \mathrm{~d}$ (Table 2). AR activity in lenses of sand rats fed with acarbose for $39 \mathrm{~d}$ was less than half that of the group fed on an acarbose-free diet. Compared with untreated sand rats, the decreased AR activity of treated sand rats was accompanied by an almost threefold higher lens protein level and an almost twofold higher lens GSH content (Table 2). Acarbose had no 
Table 2. Effect of acarbose on aldose reductase (EC 1.1.1.21) activity, protein level and reduced-glutathione (GSH) content in lenses of sand rats (Psammomys obesus) treated or untreated with acarbose for $39 d$

(Mean values with their standard errors)

\begin{tabular}{|c|c|c|c|c|c|c|}
\hline \multirow[b]{2}{*}{ Treatment } & \multicolumn{2}{|c|}{$\begin{array}{l}\text { Aldose reductase activity } \\
\text { ( } \mu \text { mol NADPH/mg } \\
\text { protein per min) }\end{array}$} & \multicolumn{2}{|c|}{$\begin{array}{l}\text { Protein } \\
(\mu \mathrm{g} / \mathrm{mg} \text { tissue })\end{array}$} & \multicolumn{2}{|c|}{$\underset{(\mu \mathrm{g} / \mathrm{g} \text { tissue })}{\mathrm{GSH}}$} \\
\hline & Mean & $\mathbf{S E}$ & Mean & SE & Mean & SE \\
\hline (-) Acarbose & \multicolumn{2}{|c|}{$(n 9)$} & \multicolumn{2}{|c|}{$(n 9)$} & \multicolumn{2}{|c|}{$(n 8)$} \\
\hline (+) Acarbose & $3 \cdot 5 * * *$ & 0.55 & $240^{* * *}$ & $23 \cdot 5$ & $645^{* *}$ & $71 \cdot 1$ \\
\hline
\end{tabular}

Mean values were significantly different from those for the group without acarbose, ${ }^{* *} P<0.01,{ }^{* * *} P<0.001$.

effect on the sand rats' body weight compared with the control group, throughout the experimental period (results not shown).

\section{DISCUSSION}

Acarbose was found to decrease daily plasma glucose profiles in sand rats (Clissold \& Edwards, 1988; Madar, 1989; Madar \& Hazan, 1993a). The present findings, corresponding to those of previous studies (Kalman et al. 1993; Madar \& Hazan, 1993b), indicate that apart from reducing postprandial hyperglycaemia (Madar \& Hazan, 1993 a, b), acarbose lowers plasma glucose levels throughout the entire day in sand rats. These results clearly show the potential benefit of acarbose in controlling plasma glucose in the sand-rat model.

Acarbose prevented or delayed cataract development in sand rats as shown in the present study (Figs 2 and 3). This beneficial effect of acarbose was also seen when the location and histopathological changes were evaluated. As acarbose is only scantily absorbed in its active form (1-4\%) from the gastrointestinal tract into the systemic circulation (Krause $e t$ al. 1993), it is reasonable to assume that its inhibitory effect on cataract development is indirect rather than direct, via the reduction of daily plasma glucose profile. The association between hyperglycaemia and cataract development is well established (Kador et al. 1986; Kinoshita, 1986). In addition, acarbose feeding prevented depleted protein and GSH levels in the lens whereas in the untreated group the hyperglycaemic state was accompanied by rapid cataract development, elevated lens AR activity and depleted lenticular protein and GSH levels.

Much attention has been focused on decreased antioxidant levels with respect to cataractogenesis, particularly on the loss of GSH (Bunce et al. 1990; Ohrloff et al. 1994). GSH is among the major aqueous antioxidants in the lens (Taylor, 1989).

The enzyme glutathione reductase $(E C$ 1.6.4.1) is necessary for maintenance of the cellular pool of GSH. Since glutathione reductase and AR compete for NADPH in the lens (Chang et al. 1983; Giblin et al. 1983), we assume that NADPH depletion by AR causes decreased glutathione reductase activity with resultant depletion of lenticular GSH levels (Gonzalez et al. 1983; Lee et al. 1985). Consequently, the lens may be more susceptible to damage by free $\mathrm{O}$ radicals (oxidation stress) since $\mathrm{GSH}$, like other antioxidants, plays a significant role in protecting against oxidative stress (Hothersall et al. 1988). 
Although the mechanism of cataractogenesis has not been firmly established, three distinct trends, all arising from increased ambient glucose concentrations, combine and possibly contribute to cataract formation. These include (1) sorbitol accumulation resulting from increased AR activity; our preliminary results indicated that acarbose may lead to lower levels of sorbitol in the sand rats' lenses, (2) oxidation damage, and (3) non-enzymic glycosylation of lens proteins (Gonzalez et al. 1983).

As shown in the present study, inhibition of cataract development resulting from acarbose treatment was associated, at least in part, with the prevention of AR hyperactivity. Inhibition of lens AR could prevent osmotic damage and increase lens membrane permeability and, consequently, could prevent GSH leakage. In addition, since AR activity is not elevated, NADPH depletion may not occur and glutathione reductase activity and GSH levels may not decrease. This could further explain the effect of acarbose on lenticular GSH levels.

The fact that acarbose may prevent both osmotic damage and increased lens membrane permeability can also explain how it restores protein levels in the lens, since shutdown of protein synthesis might be prevented and proteins may not be able to penetrate the lens membrane. Further investigation should explain this phenomenon.

In conclusion, acarbose was found not only to control plasma glucose levels in diabetic sand rats, but also to prevent cataract development, visually and histologically. Furthermore, factors related to cataract development, such as AR and glutathione, were also found to be affected by acarbose. The mechanism by which acarbose led to these effects remains to be elucidated.

\section{This work was supported by Bayer AG, Germany.}

\section{REFERENCES}

Bancroft, J. D. \& Stevenes, A. (1972). Theory and Practice of Histological Techniques. Edinburgh, London, New York: Churchill Livingstone.

Bischoff, H. (1993). Pharmacology of $\alpha$-glucosidase inhibitors. In $\alpha$-Glucosidase Inhibition: Potential Use in Diabetes. Drugs in Development, vol. 1, pp. 3-13 [J. R. Vasselli, C. A. Maggio and A. Scriabine, editors]. Branford: Neva Press.

Bradford, M. M. (1976). A rapid and sensitive method for the quantitation of microgram quantities of protein utilizing the principle of protein-dye binding. Analytical Biochemistry 72, 248-254.

Bunce, G. E., Kinoshita, J. H. \& Horwitz, J. (1990). Nutritional factors in cataract. Annual Review of Nutrition 10, 233-254.

Chang, H. M., Fagerholm, P. \& Chylack, L. T. J. (1983). Response of the lens to oxidative-osmotic stress. Experimental Eye Research 37, 11-21.

Clissold, S. P. \& Edwards, C. (1988). Acarbose: a preliminary review of its pharmacodynamic and pharmacokinetic properties, and therapeutic potential. Drugs 35, 214-243.

Diabetes Control and Complication Trial Research Group (1993). The effect of intensive treatment of diabetes on the development and progression of long-term complications in insulin-dependent diabetes mellitus. New England Journal of Medicine 329, 977-986.

Giblin, F. J., McCready, J. P. \& Reddy, V. N. (1983). The role of glutathione metabolism in the detoxification of HO in rabbit lens. Investigative Ophthalmology and Visual Science 22, 330-335.

Gonzalez, A. M., Sochor, M. \& McLean, P. (1983). The effect of an aldose reductase inhibitor (sorbinil) on the level of metabolism in lenses of diabetic rats. Diabetes 32, 482-485.

Halder, A. B. \& Crabbe, M. J. C. (1984). Bovine lens aldehyde reductase (aldose reductase): purification, kinetics and mechanism. Biochemical Journal 219, 33-39.

Hissin, P. J. \& Hilf, R. (1976). A fluorometric method for determination of oxidized and reduced glutathione in tissues. Analytical Biochemistry 74, 214-226.

Hothersall, J. S., Taylor, C. E. \& McLean, P. (1988). Antioxidant status in an in vitro model for hyperglycemic lens cataract formation: effect of aldose reductase inhibitor. Biochemical Medicine and Metabolic Biology $\mathbf{4 0}$, $109-117$.

Kador, P. F., Akagi, Y. \& Kinoshita, J. H. (1986). The effect of aldose reductase and its inhibition on sugar cataract formation. Metabolism 35, 15-19. 
Kalman, R., Adler, J. H., Lazarovoci, G., Bar-On, H. \& Ziv, E. (1993). The efficiency of sand rat metabolism is responsible for development of obesity and diabetes. Journal of Basic Clinical Physiology and Pharmacology 4, $57-68$.

Kato, K., Nakayama, K., Ohta, M., Murakami, N., Murakami, K., Mizota, M., Miwa, I. \& Okuda, J. (1990). Effect of novel hydantoin with aldose reductase inhibiting activity of galactose-induced cataract in rats. Japanese Journal of Pharmacology 54, 355-364.

Kinoshita, J. H. (1986). Aldose reductase in the diabetic eye: XLIII Edward Jackson Memorial Lecture. American Journal of Ophthalmology 102, 685-692.

Krause, H. P., Ahr, H. J. \& Boberg, M. (1993). Pharmacokinetics and metabolism of acarbose. In Drugs in Development, vol. 1, pp. 15-23 [J. R. Vasselli, C. A. Maggio and A. Scriabine, editors]. Branford: Neva Press.

Lee, S. M., Schade, S. Z. \& Daughty, C. C. (1985). Aldose reductase, NADPH and NADP ${ }^{+}$in normal, galactosefed and diabetic rat lens. Biochimica et Biophysica Acta 841, 247-253.

Madar, Z. (1989). The effect of acarbose and miglitol (BAY-M-1099) on postprandial glucose levels following ingestion of various sources of starch by nondiabetic and streptozotocin-induced diabetic rats. Journal of Nutrition 119, 2023-2029.

Madar, Z. \& Hazan, A. (1993a). Effects of acarbose and miglitol in vivo on carbohydrate digestion in type I diabetic, zucker obese and sand rats. In Drugs in Development, vol. 1, pp. 95-107 [J. R. Vasselli, C. A. Maggio and A. Scriabine, editors]. Branford: Neva Press.

Madar, Z. \& Hazan, A. (1993 b). Effect of miglitol and acarbose on starch digestion, daily plasma glucose profiles and cataract formation. Journal of Basic Clinical Physiology and Pharmacology 4, 69-81.

Marquie, G., Duhault, J. \& Jacotot, B. (1984). Diabetes mellitus in sand rats (Psammomys obesus). Diabetes 33, 438-443.

Morrison, B. (1972). Use of the Beckman glucose analyzer for low and high glucose values. Clinica Chimica Acta 42, $192-194$.

Ohrloff, C., Hockwin, O., Olson, R. \& Dickman, S. (1994). Glutathione peroxidase, glutathione reductase and superoxide dismutase in the aging lens. Current Eye Research 3, 109-115.

Taylor, A. (1989). Associations between nutrition and cataract. Nutrition Reviews 47, $225-234$.

Taylor, R. H. (1991). Alpha-glucosidase inhibitors. In New Antidiabetic Drugs, pp. 119-132 [C. J. Bailey and P. R. Flatt, editors]. London: Smith-Gordon Co.

Zimmerman, B. R. (1992). Preventing long-term complications: implications for combination therapy with acarbose. Drugs 44, 54-66. 